\title{
NOTE ON RELAXATION OF COOLING-INDUCED STRAINS IN TWO-PHASE AISI-ALLOYS AT ROOM TEMPERATURE
}

\author{
J.G.M. van Berkum, R. Delhez, Th.H. de Keijser, E.J. Mittemeijer, P. van Mourik \\ Laboratory of Metallurgy, Delft University of Technology, \\ Rotterdamseweg 137, 2628 AL Delft, The Netherlands \\ (Received July 24, 1991)
}

\section{Introduction}

Supersaturated AlSi-alloys, made by rapid quenching from the melt, have been aged at elevated temperatures. After precipitation of all silicon, cooling to room temperature leads to an average lattice parameter of the Al-matrix larger than the equilibrium value (1) and to lattice-parameter variations around the average value (2). These findings have been based on observations of shift and broadening of X-ray diffraction lines and have been explained successfully from the large difference in the thermal expansion coefficient, and thus in shrinkage on cooling, of the Al-matrix and the Si-precipitates. It has been shown that Eshelby's theory, originally developed for point imperfections, provides reasonable estimates of the magnitudes of the deviation of the average lattice parameter as well as of the lattice-parameter variation, both as a function of silicon content. In view of the relatively small values of thermal misfit in the specimens concerned, fully elastic accommodation of the misfit has been assumed. In particular if large values of misfit occur, plastic accommodation of (part of) the thermal misfit can be realized during cooling already (3).

Stress relaxation in aluminium films is a well-known phenomenon in integrated circuits technology (e.g.4-6) as well as in metal-matrix composites (e.g.7). It has been shown that stresses induced by thermal expansion mismatch can relax considerably within a few hours at room temperature.

In view of these observations, the measurements have been repeated on the specimens used in the original line-shift and line-broadening analyses, after twelve and four years of ageing at room temperature respectively, to assess a possible relaxation of the residual stress. Moreover, the original line broadening data have been reanalysed with the time interval between cooling and measurement as an extra variable.

Stress relaxation at room temperature is also of technical importance, since AlSi-alloys are extensively used as commercial casting alloys.

\section{Theory}

The following equation has been derived for the deviation of the average lattice parameter of an A-matrix with respect to its equilibrium (strain-free) value, caused by spherical misfitting B-particles in this matrix (1):

$$
\Delta a_{A}=4 a_{A} \frac{\mu_{A}}{3 K_{A}} C \frac{\varepsilon}{(1+\varepsilon)^{3}} y_{B}
$$

where $a_{A}$ is the lattice parameter of the matrix, $C=3 K_{B} /\left(3 K_{B}+4 \mu_{A}\right)$ with $\mu$ and $K$ denoting the shear and bulk moduli, $\mathrm{y}_{B}$ is the volume fraction of the $B$-particles and $\varepsilon$ is the linear misfit parameter. For the case considered $\varepsilon=\Delta \alpha \Delta T$, the product of the differences in thermal expansion coefficient and in temperature. Using Bragg's law, $\Delta \mathrm{a}_{\mathrm{A}}$ can be straightforwardly related to a shift of diffraction-line position.

Using the same model, an equation can be obtained for the mean square of the relative lattice-parameter variation (mean squared strain $\left\langle\mathrm{e}^{2}\right\rangle$ ) $(2)$ : 


$$
\left\langle\mathrm{e}^{2}\right\rangle^{1 / 2}=\frac{2}{5} \sqrt{5} \mathrm{C} \frac{\varepsilon}{(1+\varepsilon)^{3}} \sqrt{\mathrm{y}_{\mathrm{B}}}
$$

The root mean squared strain $\left\langle e^{2}\right\rangle^{1 / 2}$ is related to the integral line breadth $\beta$ as (8):

$$
\beta=k\left\langle e^{2}\right\rangle^{1 / 2} \tan \theta
$$

where $k$ is a constant, depending on the strain distribution in the specimen. In practice, rather arbitrarily, often $\mathrm{k}=4$ is taken. Van Mourik et al. (2) also adopted this value.

Stress relaxation will manifest itself by a decrease in line shift and line broadening. Probably the stored elastic energy, which is proportional to $\left\langle\mathrm{e}^{2}>\right.$, has to be considered as the driving force for stress relaxation. This means that the relative decrease of $\Delta a \mathrm{al}$ and $\beta$ will increase with the misfit parameter as well as with the volume fraction of B-particles (see eq. (2)). In the literature various behaviours of stress or strain in time are proposed (e.g.4,5,9). Here the time dependence will not be investigated in detail, since per specimen only two (incidentally three) measurements in time were available.

\section{Experimental}

Ribbons of AISi-alloys of different compositions have been prepared by the melt-spinning technique and annealed at elevated temperatures ( $397-448 \mathrm{~K})$ until all silicon was expected to be precipitated. Part of the specimens, utilized by Mittemeijer et al. (1), were in a sufficiently good shape to determine their lattice parameter again in the same way as 12 years ago: from Debye-Scherrer photographs using the Nelson-Riley extrapolation (see ref. (1)). Conceming the line broadening, all specimens (annealed at $447 \mathrm{~K}$ ) and data files used in ref. (2) were still available (including the reference for removal of instrumental broadening). $X$-ray diffraction measurements have now been repeated in the same way as 4 years ago (see ref. (2)). The correction for instrumental broadening was also identical, i.e. by using Voigt functions (8). Only the problem of the $\mathrm{Al}$ (331) and Al 420$\}$ reflections being overlapped by the tails of the $\mathrm{Si}(531)$ reflection, in the past solved by an educated guess (2), has been handled in an improved way for the old as well as for the new data files. Now, three splitpseudo-Voigt profiles have been fitted to the corresponding intensity data using the program PROFIT (10). The actually recorded number of counts at a certain $2 \theta$-position have been distributed over the three profiles according to the ratio of their relative intensities at that $2 \theta$-position as established by the pseudo-Voigt fits. In this way no assumption on the peak shape has been applied directly to the actual profiles. Line breadths have been determined of the separate profiles thus obtained.

\section{$\underline{\text { Results }}$}

The results of the present and of the former lattice-parameter measurements are shown in Table 1. From Table 1 it is clear that for the 12.5 vol.\% Si specimens $\Delta \mathrm{a}_{\mathrm{Al}}$, the difference between the actual value of the lattice parameter and the equilibrium value, has been reduced significantly on ageing at room temperature for 12 years. The increase for AlSi2.8 cannot be considered as statistically significant. Since the specimens containing 25.1 and $38.2 \mathrm{vol} . \% \mathrm{Si}$ are not in our possession anymore, the dependence of $\Delta \mathrm{a}_{\mathrm{Al}} v s$. $\mathrm{y}_{\mathrm{Si}}$ cannot be investigated.

TABLE 1

Deviation $\Delta \mathrm{a}_{\mathrm{Al}}$ for the Al-matrix of AlSi-alloys after Two Different Ageing Times at Room Temperature

\begin{tabular}{cccc}
$\begin{array}{c}\text { Si content } \\
\text { (vol.\%) }\end{array}$ & $\begin{array}{c}\text { precipitation } \\
\text { temperature }(\mathrm{K})\end{array}$ & \multicolumn{2}{c}{$\Delta \mathrm{a}_{\mathrm{Al}}\left(10^{-5} \mathrm{~nm}\right)$ at $293 \mathrm{~K}$ after } \\
\cline { 4 - 5 } & & $\mathrm{a}$ few days & 12 years \\
\hline 2.8 & 397 & 0.3 & 1.1 \\
12.5 & 397 & 6 & 2 \\
12.5 & 425 & 7 & 4 \\
12.5 & 448 & 7 & 2
\end{tabular}

Equilibrium value of the lattice parameter of pure $\mathrm{Al}$ at $293 \mathrm{~K}: 0.40496 \mathrm{~nm}$ (see ref. (1)). Accuracy of lattice-parameter values is approximately $1.10^{-5} \mathrm{~nm}$; for the $2.8 \mathrm{vol} . \% \mathrm{Si}$ specimen somewhat better because of sharper lines. 
The results of the old line-broadening measurements have been plotted in Fig. 1a (note that values for $\mathrm{Al}$ \{331\} and (420) differ slightly from those of ref. (2) due to the present improved data evaluation). Fig. $1 \mathrm{~b}$ shows the results of the new measurements after four years of ageing at room temperature. In both figures two theoretical lines on the basis of eqs. (2) and (3) are drawn: one for the rather arbitrary value $k=4$, the other for $k=5.2$. The latter value is suggested by recent line-profile simulations based on the model of misfitting particles in a matrix. The simulations confirmed the linearity of $\beta v s$. $\sqrt{y}_{S i}$ and provided the better value $k=5.2$ for the strain distribution under study (11).

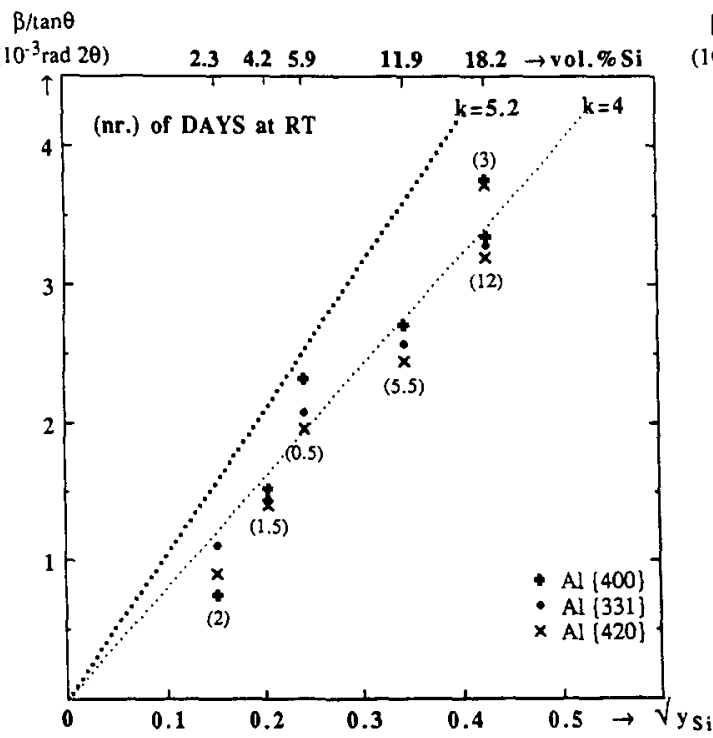

(a)

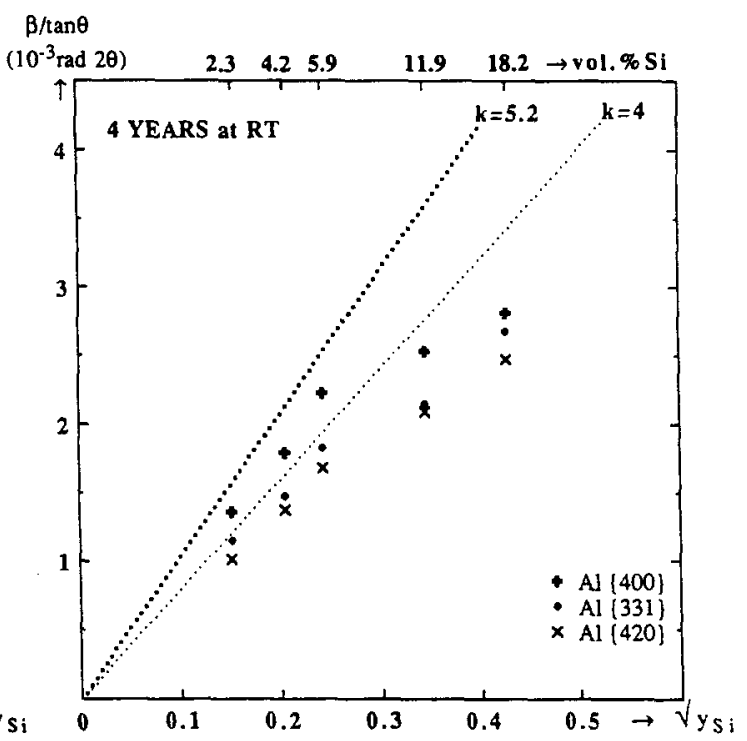

(b)

FIG.1: Normalized integral breadth, $\beta / \tan \theta$, corrected for instrumental broadening, of Al-matrix reflections vs. the square root of the volume fraction Si-precipitates, $\sqrt{y}_{\mathrm{Si}}$, after 0.5 to 12 days (a) and 4 years (b) of ageing at room temperature (RT). Straight lines of small dots are theoretically predicted values (for meaning of $\mathrm{k}$ : see text).

Compared with the theoretical line for $\mathrm{k}=5.2$, which applies to unrelaxed specimens, all data points in Fig. 1a show a smaller integral breadth, indicating that in the first few days after cooling to room temperature appreciable stress relaxation by some form of plastic accommodation has already occurred. In the time period, covered by Fig.1a, the specimen containing 18.2 vol.\% Si has been measured twice: after 3 and after 12 days of ageing at room temperature. The clear decrease in line breadth between the two sets of measurements confirms that a rapid stress relaxation was taking place. This decrease, for a single specimen, is meaningful, since only the small random errors due to counting statistics ( 1 to $3 \%$ ) have to be considered. Comparisons between data points from different specimens, however, are more difficult, since then the inaccuracies due to Si-content determination, sample inhomogeneity, insufficient flatness of the sample, etc. have to be taken into account. For this reason fitting of relaxation models to the deviations from the theoretical line in Fig. 1a did not give reliable results. Nevertheless, the 'scatter' of the data points in Fig. la seems to give a clear indication of stress relaxation. The specimen yielding the relatively largest breadth (AISi 5.9) was the first to be measured (after 10 hours), where specimens yielding relatively narrow lines were measured later (e.g. AlSi 11.9 after $5 \frac{1}{2}$ days).

The data for the three specimens richest in Si, shown in Fig.1b, confirm that continued stress relaxation can take place on ageing at room temperature again. The specimen AlSi 18.2, for example, showing on average $84 \%$ of its theoretically predicted breadth after 3 days and $74 \%$ after 12 days (see Fig. 1a), has decreased to $60 \%$ after 4 years (see Fig.lb). 
The clear downward curvature of $\beta v s$. $\sqrt{ }_{\mathrm{Si}}$ after 4 years of ageing at room temperature may be understood if the total stored elastic energy in the specimen is the driving force for stress relaxation (see below eq.(3)). The relative decrease in $\beta$ is largest for the specimens richest in $\mathrm{Si}$.

Surprisingly, the specimens containing 2.3 and 4.2 vol.\% Si show a somewhat larger line breadth after 4 years of ageing at room temperature (see Fig.1b), as compared to a few days (see Fig.1a). It has to be realized that errors due to correction for instrumental broadening are relatively large for these data points (net, structural, broadening is smaller than the instrumental broadening). If the increase is a real structural effect, it may be caused by a continuation of precipitation at room temperature. From Fig.5 in ref.(12), one would expect no increase in lattice parameter (and, thus, in line breadth) after $1841 \mathrm{~h}$ at $448 \mathrm{~K}$ for a liquid-quenched specimen containing 2.3 at.\% Si $(=2.8$ vol.\%). The precipitation in ref.(12), however, may have been accelerated by the manifold cooling and heating, necessary for the lattice-parameter measurements: every cooling is followed by stress relaxation at room temperature, causing less resistance against further precipitation at the annealing temperature. This is especially important in the low Si-content specimens, where the diffusion distances in the final stage of precipitation are largest, realizing that the precipitate sizes are approximately independent of the overall Si content (1). Then, the small increase of the Al-matrix lattice parameter of the specimen containing 2.8 vol.\% Si on ageing at room temperature (see Table 1), after the precipitation treatment of $512 \mathrm{~h}$ at $397 \mathrm{~K}$, can also be understood as the result of continued precipitation.

The $\{400\}$ reflection is clearly more broadened than the $\{420\}$, which in turn is more broadened than the $\{331\}$ (see Fig.1)*. This order of the normalized breadths $(\beta / \tan \theta$, see eq. (3)) of the 3 reflections is not equal to the order in 20-position. Therefore it is not likely that the differences are due to instrumental effects. It is suggested that the elastically anisotropic behaviour of the Al-matrix explains the observations. As for most cubic materials, Young's modulus increases with the sum $h^{2} k^{2}+k^{2} 1^{2}+h^{2} 1^{2}$, where $h, k$ and $l$ are the indices of the unit vector normal to the diffracting planes, corresponding to the reflection $\{h, k, l\}(13)$. This sum takes the values $0,0.16$ and 0.27 for the $\{400\},\{420\}$ and $\{331\}$ respectively. As Young's modulus increases, the elastic compliance of the lattice, and thus the strain, will decrease, as observed in Figs.1a and 1b.

\section{Conclusion}

The difference in thermal expansion coefficients of precipitate and matrix in two-phase AlSi-alloys causes non-uniform strains, which result in an increase of the average lattice parameter and of the lattice-parameter variation around the mean value. An appreciable relaxation of these strains on ageing at room temperature is observed. This results in decreased X-ray diffraction line shift and line broadening after 12 and 4 years respectively. A large fraction of the misfit strains is already accommodated in the first few days after cooling to room temperature. Stress relaxation is more pronounced as the overall Si content increases, which is to be expected if the stored elastic energy is considered as the driving force for relaxation.

\section{References}

1. E.J. Mittemeijer, P. van Mourik, Th.H. de Keijser, Phil. Mag. A, A42, 1157 (1981).

2. P. van Mourik, Th.H. de Keijser, N.M. van der Pers, E.J. Mittemeijer, Scripta Met. 22, 1547 (1988).

3. M. Taya, K.E. Lulay, D.J. Lloyd, Acta Met. 39, 73 (1991).

4. M. Hershkovitz, I.A. Blech, Y. Komem, Thin Solid Films 130, 87 (1985).

5. M.A. Korhonen, C.A. Paszkiet, R.D. Black, Che-Yu Li, Scripta Met. 24, 2297 (1990).

6. J.B. Cohen, Adv. X-ray anal. 33, 25 (1990).

7. P.J. Withers, D. Juul Jensen, H. Lilholt, W.M. Stobbs, in Proc. ICCM VI, vol. 2, ed. by F.L. Matthews, N.C.R. Buskell, J.M. Hodgkinson, J. Morton, p. 2255, Elsevier Applied Science, London (1987).

8. R. Delhez, Th.H. de Keijser, E.J. Mittemeijer, Fresenius Z. Anal. Chem. 312, 1 (1982).

9. J.K. Solberg, H. Thon, Scripta Met. 19, 431 (1985).

10. J.I. Langford, D. Louerr, E.J. Sonneveld, J.W. Visser, Powder Diffr. 1, 211 (1986)

11. J.G.M. van Berkum, R. Delhez, Th.H. de Keijser, E.J. Mittemeijer, in preparation.

12. P. van Mourik, Th.H. de Keijser, E.J. Mittemeijer, in 'Rapidly Solidified Materials', Conf. Proc. ed. by P.W. Lee and R.S. Carbonara, p. 342, ASM, Metals Park, Ohio (USA) (1986).

13. J.F. Nye, Physical Properties of Crystals, p. 145, Clarendon Press, Oxford (1972).

* The (400) of AlSi 2.3 in Fig.1a is the only exception. This may be caused by inaccuracy of the measurement or the correction for instrumental broadening (the net, structural broadening is the least of all). 